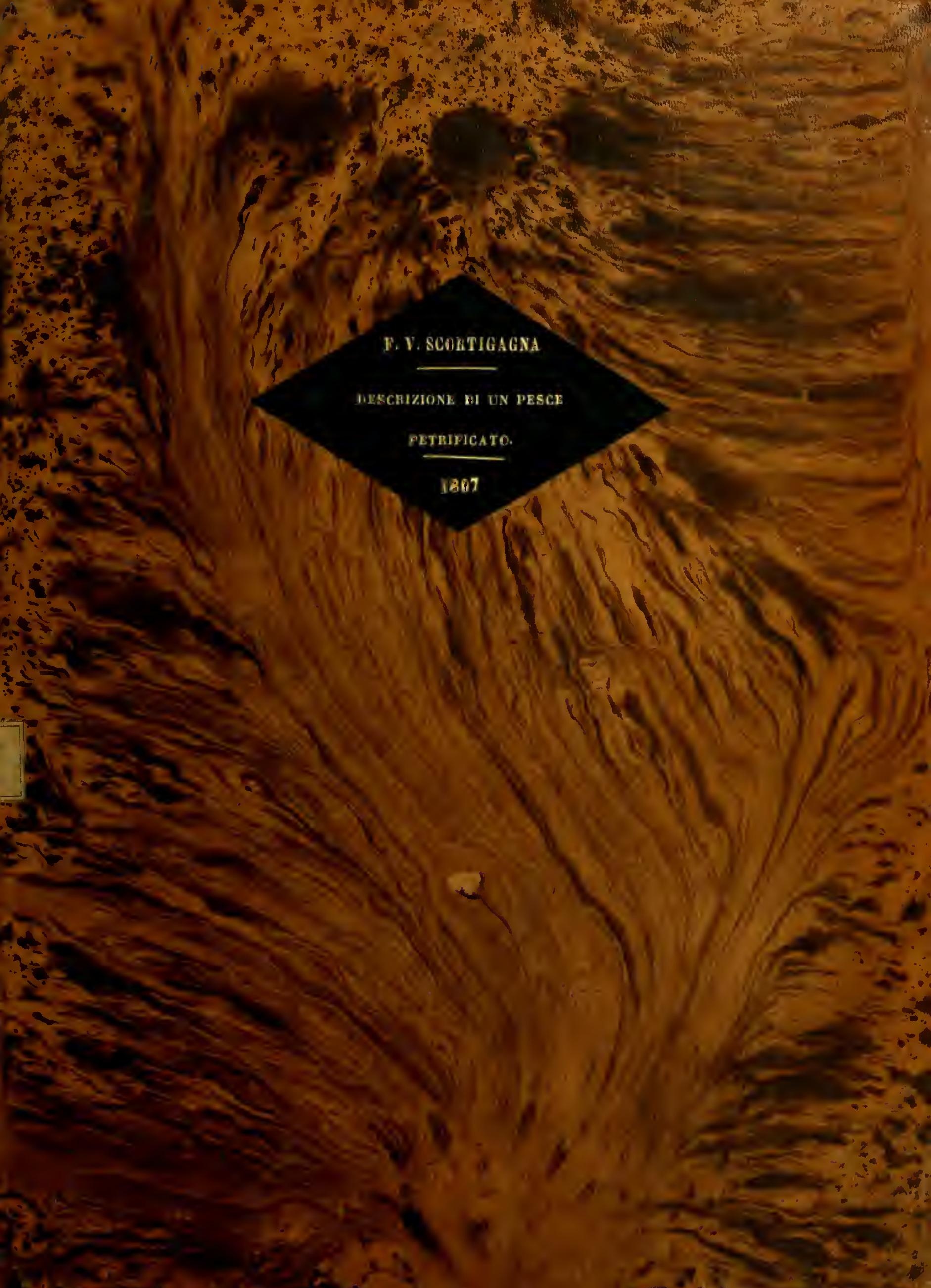




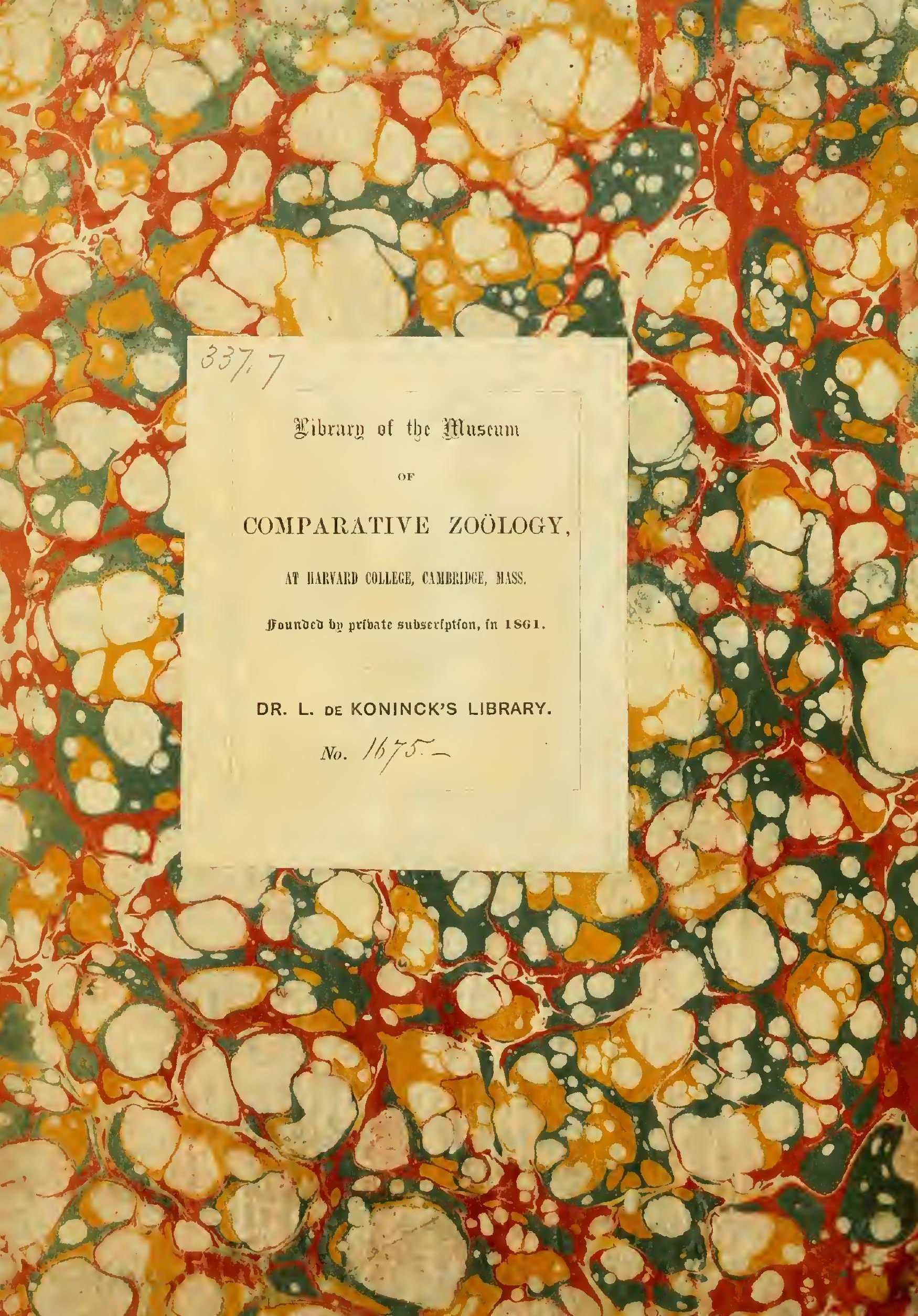







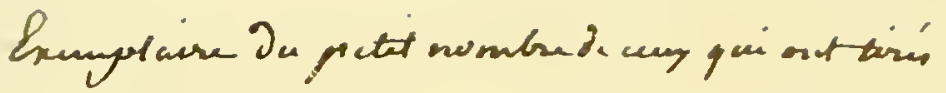
bourgreme papicer. 


$$
\because \text { Ycortegagna }
$$

A MONSIEUR

\title{
FAUJAS-DE-SAINT-FOND
}

\author{
PROFESSORE \\ AL MUSÉUM DI STORIA NATURALE \\ D I FRA N I A

\section{MEMORIA EPISTOLARE}

PER SERVIRE DI SEHIARIMENTO

\section{ALLA DESCRIZIONE DI UN PESCE PETRIFICATO}

SCAVATO IN ALTISIMO

NELLE VICINANZE DI BOLCA.

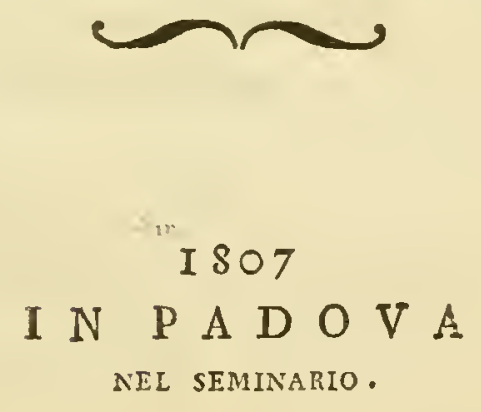


Hoc opus, hoc studium parvi properemus, dor smpli. Si patria volumis, si nobis vivere cari.

Horat, Epist, III, Lib, I. 


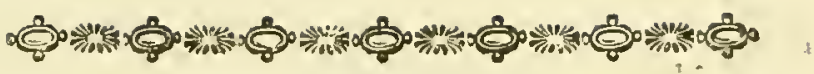

$P_{\text {ER maggior intellfgenza di quefta memoria cre }}$ diamo neceffario di far conofecre due altri opufcoletti volanti, che ufcirono alla luce intorno l'ittiolito di cui qui fi tratta, e delli quali non venne fatto parola nel noftro giornale. Il primo è una lettera del fig. dott. Francefco Orazio Scortigagna all' ornatiff. co: Arnaldo Turnicri : quefta lettcra fcritta nelle dae lingue italiana e latina prefenta una fuccinta ftoria della foperta di quefto bel petrefato; ed c̀ divifa in cinque paragrafi.

Nel primo paragrafo l'Aut. riffettendo a quento ittiolito lo confidera degno d'ogni nobile gabinetto, non che lo caratterizza argomento ben degno d' cffere defcritto dai dotti ed illuminati ittiologifti, il che non effendo ftato per anche efeguito ha egli creduto ben fatto il tefferne la defcrizione nel miglior modo che per lui fi poteffe.

Nel fecondo arverte di averne fatto il difegno;

A 2 di 
di cui ne ha porto la tarola in fine del libro; che rapprefenta l'ittiolito in grandezza della duodecima parte dell' originale.

$\mathrm{Nel}$ terzo egli indica la pictra in cui racchiudefi, cioc in una pictra calcarc-argillofa-fchifto$r a$, di cui porge $i$ caratteri, e prende in efame lc parti più offervabili c le principali notc caratteriftiche dcll'ittiolito, c fono il fuo colorito, la fua tefta $c$ denti, le duc macchic circolari alla fuperiore fezione della tefta, le vertebre, la coda colle fuse accidentalità, la fua giacitura, una fola pinna dorfale, la pinna, offia appendicetta caudale, due pinne pettorali, la diftanza dell' apice della coda, l'cftemiti del fuo mufo, finalmente la fua totale eftenfione; dalle quali riffefioni,

$\mathrm{Ncl}$ quarto ftabilife il gencre dell' ittiolito effere quello del cane marino, Squalus Linnei pel talc qualificato anche da alcuni pefcatori di mare. che ritrovandofi cafualmente culì, furono cfil per il natural genio portati a vederlo.

Ncll' ultimo paragrafo ftabilifee dubitativamente la spezie cffere cane renardo di mare, ciò deducendo dalla mancanza delle pinne addoninali, dalla pinna dorfale, dalla qualitì dclla fua polle piuttofto lifcia e zigrinata, e finalinente dalla forma della tefta dilatata, c conchiude con tutta modcftia non poterfi chiamare crrore it crederlo profímamente fimile alla fopraddetta fpezie delli renardi 
marini; e ciò fino' a tanto che migliori lumi "acquiftar fi poffano fopra tale argomento, concludendo di aver ardito dirne più di quello egli poteva, come mancante di mezzi e di cognuzioni atte a qualificare fenza equivoco l'indicata fpezic.

L'altro.fcritto è una lettcra del conte Gazola ufcita in Verona l'anno 1 So 5 . dalla tipografia Tomafi diretta al fuddetto lig. dott. Francefco Orazio Scortigagna di Lonigo, la quale fi pirò confiderare divifa in due parti. Nclla prima fi tratta della ftoria, della fcoperta e delle inforte queftioni pel diritto di proprietà ma ficcome quefte piccole brighe fono argomento quanto importante per il particolare altrettanto indifferente per l'univerfalc, e di niun profitto per la feienza, cosi bafti averne qui fatto cenno. La fcconda parte poi contienc una deferizione $c$ determinazione del pefee differente da quella pubblicata dallo Scortigagna nella lettera al co: Tornieri fopra indicata. Ora in quefta deferizione del Gazola ii dice, che il pefee non è nuovo alle cave bolcane, ed alcuni cfemplari in quelle efcavati fi confervano nel mufeo di Nimes (10) di Parigi c nel gabinetto del detto co: Gazola, c puofene vedere la defcrizione (II) nell' Ittiolitologia vero- 
veronefe gial da qualche anno incominciata a fortire dai celcbri torchi giuliariani. La forma del corpo, la pofizione delle pinne, la figura della coda e dei denti lo annunziano fenza dubbietà per lo fqualo cane carcaria di Linnco (1 2), Bellonio (13), Gefner (14), Aldrovandi (1j), Jonfton (i6), e dcil'Enciclopedia. In quelta fi trova definito il cane carcaria

S. carcharias dorso plano; dentibus ferratis. L'ampiezza del dorfo è ben facile a fupporfi dalla figura annetra alla defcrizione del fig. Scortigagna, febbene non fi polfa, attefo il diffeccamento e la compreflione del pefce, riconofcere la fuperficic lar ga e piana. Non corrifponiono ad una tale deferizione $i$ denti, che non fono addentellati a modo di fega, ma siò nulla ofta al coftituir!o nella fpezie anzidetta, qualora vogliali preftal fede al fempre celebie Linnco, che lo fqualo carcaria è (17) Sex (juniori paucioribus) dentium, frequenter fofflium, triangularium, (juniori nondum ferrotorum) ordinibus borrens.

Gronovio ( 18 ) aggiunge agli altri caratteri anco quecllo

(12) Loco cit. p. I 494, n, I2.

(13) Aq. p. 58.

(14) Aq. p. 173 .

(15) Pisc. p. $381,382.3870$

(i6) Pisc. p. $24, t .6$. f. 6.

(I 7 ) Loco citato.

(18) Mus, I, 138, zooph. 143 . 
quello delle pinne pettorali grandifime: pinnis peAtoralibus maximis, e tali certamente fono quelle clie il fig. Scortigagna ha moftrate nelia fua tavola. La pinna dorfale rotondata quali a perpendicolo della pinna dell'ano, c̀ rifcontrata dal detto fig. Francefco Orazio Scortigagna, e forma deffa ugualmente un carattere del carcaria. Le ventrali fono diftrutte, forfe perchè efili, effendo d' affai tenue fibratura: ma accennano certamente (non dubitando dell' cfattezza del difegno già indicato) i sudimenti nel luogo donde fortivano.

Dal fin qui detto rifula certamente, che più affai che fra' renardi, o volpi marine abbia ad annoverarfi ginefto ittiolito fra i cani carcaria.

Oltre di che per reftare perfuali delle cofe dette non farà fuori di propolito il fentire l'opinione del più celebre fra gl' ittiologhi viventi il fig. Lacépede (21). Egli nclla fuperba e ricca opera, che gli meritò il titolo di cmulo dell' immortale M. di Buffon, e che fi conferva dal fig. co: Gazola a monumento preziofo dell' amicizia di cui è onorato, ci dà la più efatta deferizione dei cani carcaria fra quante n'abbia avute fin qui la ftoria ittiologica .

"Lc corps du requin eft très-alongé, \& la

" peau

(21) Loco cit. c. 174 , 
" peau qui le recouvre en garnie de petits tu"bcrcules très-ferrés les uns contre les auties ,. Ecco il corpo allungato qual vedefi nella figura della defcrizione e la pelle zigrinata, che dal fig. Scortigagna ad evidenti riprove fu rifcontrata nel fuo renardo.

"La tête elt aplatie, continua il dotto la Ce" pède, \& terminéc pal un mufeau un peı al"londi ". Schiacciata c̀ la tefta del pefce difegnato nclla detta tavola del fig. Francefco e rotondata; ma ciò mal confronta coi caratteri del pietefo renardo, che lo fi vuole dall' Enciclopedia di una tête d'une forme conique, $c$ da Linnco capite conico, brevz, mentre affai bene confionta col cane carcaria. La compreflione cagionata dalle fovrincombenti materic nel tcmpo dell'accaduta petrificazione, come fi accenna a c. Is della detta lettera, milita più a provare che il poco rotondo fia divenuto molto, piuttofto che fiafi il conico rotondato.

"La forme de la bouche cft en forme de "demi-cercle, \& placée tranfverfalement au def"fons de la tête $\&$ derricre le narins,. Qucfto carattere conviene a perfezione.

"Elle eft très-grande, \& l'on pourra juger "facilcment de ces dimenfions, cn fachant, que "nous arons reconnue d'après pittlieurs comparai"fons, que le contour d'un côté de la machoire fupé- 
;2 fupéricure; mefuré depuis l'angle de deux ná" choires; jufqu'au fommet de la machoire d'en"haute, égale à peu-prìs le onzieme de la lon„gucur totale de l'animal „. Prefa dalla detta figura la mifura deferitta, il più clattamente porfibile, fi trova con precifione corrifponderc la lunghezza totale del corpo del pefce già deferitto dal fig. Scortigagua alle undici dimenfioni accennate. Qual prova maggiore puoffi mai defiderare della prefente, e come unita all'altre non fi ftabilirà nclla fpezic de' cani carcaria il pefec dal fig. Francefco ripotato?

Riman folo a provarfi, ch' effo foffe ancor fanciullo per togliere anco l'obbiezione de' denti non dentellati a foggia di fega. Ma ciò è tofto fatto qualor is fappia che liffatta fpezie giunge fino alla lunghezza di 25 e 30 piedi parigini. Nc̀ ciò deve forprendere, fe prestili fede a Gunner (21), che racconta efferfi trovato un vitcllo marino della groffezza di un bue nel ventre d'uno di quefti pefci; a Mulcr (22), che afferifce che un cavallo intero, ch'cra ftato gettato da un baftimento, liafi rinvenuto negl'interiori di un carcaria pefcato nell'ifola di S. Margherita, il di cui pefo era lib. I 500 ; a

(2I) Pront. Schiften Tom. II. pag. 301.

(22) Tom. III. p. 267 . 
10

Bruniche (23) teftimonio della fezione d'un altro

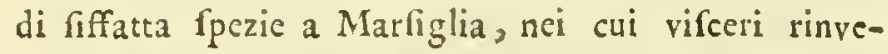
nironfi due grofil tonni ed un uomo tutto veftito; come del pari afferifec il Rondelezio (24), c col racconto di fatti analoghi confermano Cetti (25), Fermin (26), il Padre Fevillé (27), e l'immoital Bloch (29).

Quale farà ftata la patria di quefto ittiolito? Se il fi vuole un renardo o volpe inarina, convengono tutti gli Autori, ch'effo tia abitatore del mediterraneo fino al mare di Scozia; fe poi, come crede il fig. co: Gazola di non mal apporfi, $\grave{c}$ un cane carcaria è affai conofciuto, che tutto l'oceano gli è patria ed abitazione.

L'eftratto ch'abbiamo dato di quefti due opufcoli faciliterì ai noftri letrori l' intelligenza della feguente memoria epiftolare, e li metterà in fituazione di poter dar ful contenuto della inedefima un più fondato giudizio.

Chia-

(23) Reis nach der Vorgeb der guten Hoffnung p. 374 .

(24) Hist. des poiss. P. I. p. 306. 
Chiarifimo fig. profeffore.

§. I. Egli ¿̀ omai tcmpo, clie adempiafi per mia parte ciò che da più meli, o chiariflimo fig. profeffore, cbbi l'unor di promettervi. Se in ciò efeguirc s"interpore dilazione sì lunga, li dovrà incolparne le peculiari circoftanze che mi li oppofero, non: mai la volonterofa mia difpolizione.

Il pefce foffile da me compendiofamente defcritto con foglio (1) 26. maggio $1 \$ 05$. non merica per certo d'effere pofto in obblio, e ricerca altresi ulteriori efami intorno alla. fua fpezie, tanto più che una lettera ftampata (2) in data 1. agofto I so 5. del fig. Gio: Battifta. Gazola di Verona a sne diretta, mi mette in dovere di faclo; in vifta di che, ommettendo efpreffamente di rifpondere a quanto nella fuddetta lettera apparife fcoftarli dagli oggetti fcientifici, mi affretto di paffare agli fchiarimenti dell' accennato compendiofo mio foglio.

\$. II. Nell' introduzione del medelimo io non fecí che un cenno (3) in propofito della difficoltit dellu

(1) Ved. Descrizione di un pesce pietrificato ec. Vicenza tipografia Parise 1805.

(2) Ved. Lettera al sig. Francesco Orazio Scortigagna di Lonigo sopra $l_{2}$ descrizione di un pesce pietrificato ec. Verona stamperia Tomasi .

(3) Ved. Descrizione citata pag. 6. 
18

delo ferivere qualche cofa di propofito fopra tale argomento, difficoltà che richiede di effere fentita. Se fi confideri un pefce, la cui maggiore groffezza avuto aveffe per ogni fenfo il fuo maggiore diametro di circa otto pollici ( 2 I centimctro e 6 millimetri) ridotto quafi alla fottigliezza di una carta, come appunto quello è dell'ittiolito, di cui fi parla, affermare dovrebbonfi notabilmente alterate le fue proporzioni. Le vertebre fteffe variamente curvate nella lor ferie, le une dall' altre più o ineno fcoftate, ovvero più o meno approffimate fi mirano, in forza che $i$ loro legamenti non equabilmente o fi contraffero, o per varie cagioni furon diftratti nel tempo del di lui impictrimento, o nell' epoca ad effo vicina. Un pefce morto in atto di fofferire violenti contrazioni di corpo indicate dalla fua giacitura, può facilınente avere alterato il fuo fifico per mentire una fpezic * - per mafcherarla fotto altre apparenze. Un pieirificato finalmente fu cui furon dall' arte operate le dette innovazioni non può (4) nel cafo noftro lafciare fondate fperanze per effere definito coa ficurezza.

\$. III. Ad ugigerro di rogliere gli equivoci permettetemi fig. una digreffione: digreffione ben necer-

(4) Ved, Descrizione cit, pag, s4, 
ceffaria per far conofcere, che quefto noftro petres fatto fembra non effere riferibile alla fpezie dello fqualo carcaria, o vogliam dirlo piì propriamente nella italiana favella alla. fpezic del pefce lamia, come fe ne moftra perfuafo il fig. co: Gazola o Pafferò pofcia ad indicare la fua fomiglianza od approffimazione con alcuna delle cognite fpezic aggiungendovi altre riffefioni. Reftringerafii cosi a due fole parti quefta inemoria, che mi fo un prea gio di tributaryi.

\section{P A R T E I:}

6. IV. Per foddisfare al mio affunto con mag: giore agiatezza procederò con metodo inverfo. Prenderò a confiderare primamente le parti pofteriori del pefce medefimo, per poi falire all efame delle anteriori, nelle quali affai fiù che altrove parmi rifplendere il vero della prima di dette due prow pofizioni. Me ne anderò paffo paffo ragionando dietro gl'infegnamenti del chiarifimo voltro cona fratello Monf. de la Cepède, il più eccellente di quanti altri lino qui abbiano la.. ftoria dei pefci defcritta; il quale perciò meritevolmente rifcuote 12 più ferma fingolar fede nell efattifime fue fpos fizioni .

§. V. Lo fqualo carcaria ( per valermi delle. 
parole di detto chiar. Autorc) c̀ il prototipo (s) dcll'inticra famiglia. Egli è lo fqualo per eccellenza che va ad effere il modello gcuerale, a cui poter riferire le altre fpezie. Ottima cofa dunque farà quella di prendere quefta fpezic per punto di confronto col noftro petrificato.

§. VI. Se attentamente fi offervi la bellinima figura in raine pubblicata nell' egregia opera or mentovata, il carcaria porta la coda in due lobi divifa, il fuperiore dei quali dotato di lunghe cartilagini, formanti la fua pinna, vedefi molto incurvato, c prolungato nel fuo fininento (6) da una piccola triangolare appendice. Non volendo per ora ulteriormente trattenermi nell' efame di certe varictà che nella coda s'incontrano delli carcaria - per l'età, o per il feflo, o per qualifia altra cagione diverfi, preferifco attenermi al raine accennato .

La coda del noftro ittiolito, quantunque anche effa divifa in due lobi, è ben diverfa da quella in ciò che le marginali cartilagini del lobo catidale fuperiore fono breviffinc: e la fua piccola

(5) Ved. Histoire naturelle des poissons par le citoyen la Cepède membre de l'instit. national, \& professeur au muséum d'histoire naturelle à Paris chez Plassan 1798. Tom. I. pag. $x y 2$.

(6) Ved. M. de la Cepède opera cit. Tom. I. pag. 169. \& Pl. 8. 
appendice fi offerva nun già in continuazione della coda fleffa, ma riroltata all' infù colla notabilirfima circuftanza di effere fituata pollici 2 , linee 7 ( 72 millimetri) diftante dall' cstemo apice della fua coda, come fi vede nella I. figura (7) della yui in fine anneffa tavola. La fua direzione fuperiormente riguardante del fuo rovefciamento pes varic cognizioni forfe accaduto, potrebb' effere una prosa ben convincente.

\$. VII. Il lubo inferiore della coda (S) del cone carcaria à confiderabilmente largo, ha origine

co-

(7) Il presente disegno in parte tratto dall' archetipo, ed in parte dal disegno alla naturale grandezza eseguito da diligente delineatore ha il vantaggio di esibire li contorni tanto delle parti interiori, quanto dell' esteriori dell' itticlito il più esattamente possibile. Resta pertanto invitato all' esane dell'originale stesso chi avesse dubbio in contrario. Tale esortazione vienc fatta altresi a chi avrà l'opportunità di vederc la stampa del rame, che per decreto di Governo 24 . febbraro $1 \$ 06$. N. $\frac{284}{270}$ arti e mestieri N. 7 , e per altro relativo decreto 3. marzo i $\$ 06$. N. $\frac{38 z}{43 .}$ dalla vicentina Municipalità è prossima a pubblicarsi dell'ittiolito medesimo. L'osservatore ammirerà in quella l'ottimo effetto dell' ombreggiamento tale, qual' esigono che sia le leggi della prospettiva, nonchè la gradazione delle sue tinte. In questo piccolo rame sperasi che l'intendente aggradir possa la dilucidazione dei contorni delle parti piti interessanti si esterne, che interne puramente marcate per la più facile intelligenza di quanto si tratta nella presente memoria.

(8) Ved. M. de la Cepède op. cit. loco cit. lo squalo carcaria esistente nel pubblico gabinetto di storia nat. di Pavia porta la coda in due lobi divisa, ed ha le sue pinne molto più intrecciate ancora del testè citato. 
comune, e s'innetta sol fuperiore terminando con apice rotondato.

Il lobo inferiore della coda dell' ittiolito ha forma triangolare piì riftretta, non fi ftacea dal fuperiore che a 2 pollici e 6 lince ( 65 millimetri) dalla vilibilc fua origine (9) colle cui cartilagini non molto s'intrcccia $\rightarrow$ e termina con acuta cftemità .

§. VIII. La pinna dorfale pofertiore in luogo di cffere confiderabilmente piccola, come nel carcaria, moftra invece nutabile lunghezza, fe però dir non rogliafi che quefta pinna in pria fratturata, e quivi (:0) inneftata, fituarfi invece aveffe doruto in $m$ della citata figura $I$. dove ultimamente fcoprifi il rudimento di altra pinna.

\$. IX. Ie due pinne dell'ano, e le due ventrali fono deficionti nell' ittiolito, delle quali nemmeno forgonfi li rudimenti.

§. X. Lc due pettorali efiftono, ma una di quefte, come ho (II) altra volta rimarcato, inneftata dall'artcfice nel fito, in cui mirafi in $\mathrm{N}$. fig. I., potrebb'efferc inal collocata, c forfe avreb-

(9) Ved. fig. I. La lettera Q. serve a marcare quel sito della schiena nell' ittiolito, che può corrispondere alla fossetta triangolare accennata da M. de la Cepède opera ci.. pag, 268. parlando dello squale renard.

(so) Ved. fig. I. Lettera I.

iI) Ved. la citata mia descrizione pag. 14, nota (e). 
be potuto appartenere ad altro individuo. Dalla forma peraltro, e dal tito della pinna pettorale 2. fig. I., fu cui non cade alcun dubbio, puoffi conchiuderc della forma c fituazione dell' analoga. Accordatagli pertanto un'altra pinna fimilc a quella marcata 2., rifultano le dette pinne efferc più actlminate nel loro finimento, e piì anteriormente fituate di quelle dello fqualo carcaria, che redefi nell'opera citata di M. de la Cepède.

§. XI. La pelle eminentenente zigrinata, oflia per valermi dell' efpreffione di $M$. de la Cepède (che riverentemente onorn e nomino) la pelle fornita di piccoli tubercoli ftrettamente legati gli uni contro gli altri, forma uno dei caratteri dclli carcaria. Quefto carattcre non è efclufivo ad altre fpezie, c però d̀ inconcludente farne per ora il foggetio dell' argomento, rifervato efpreffamente alla feconda parte di quefta memoria. Dall'altro canto ¿ baftantemente noto attribuirfi il nome di zigrino ad alcune altre pelli di fquali. Io non mi eftenderò nclla tcoria di quefto utilifimo ogyetto di commercio non fervendo al prefiffo mio fcopo.

§. XII. Eccomi giunto fenza avredermene alla parte la piì cnigmatica del noftro ittiolito, rogiio dirc la tcfa. Il contorno di quefta fembra avere qualche analogia col capo di quello fqualo, che nominafi il barbuto, ma differife dal medefimo per gli altri caratteri. Son avenio però con il 
18

capo degli altri fquali raffomiglianza; potrebbefi fatto rifleffo alla fua equivocitì, un talc articolo trafandare, ma mi rifervo a parlarne di propolito nella feconda parte che verrà in reguito.

§. Xill. La privazione dei laterali fori branchioneghi nell'itsiolito militerebbe piuttofto contro che in favore della fpezic delli carcaria. Potrebbero non pertanto cfli fori aver efiftito nel pefce vivente, ed cfferfi nella fua petrificazionc obbliterati. Ma io baftantemente notai, che quefto porta alla Superiore fezone della tefa due macchie circolari (12). ( $E$ non è il prefente ittiolito re non che la metà dell' intiero, cffendofi dai minatori infranto l'incontro fuo, e pofcia fimarrito; le fole vertebre aderenti quafi onninamente rimafero a quefto ben confervato efemplare). Ora quefte due macchie, od impronte clic s'accoltano alla figura circolare, ma che non lo fono perfcttamcute partecipando dell'ellitica, non parlano effe cloquentiflinamente? A me fembra che si ; poichè fe 1 'anteriore rapprefenta la membrana ammicante (clignotante dei Francefi) combina la incdefima a marcare uno degli occhj; e fe l'altra corrifponde ad uno (13) dei fori fpirali, e fe talc fi folle

Atata

(12) Ved. descrizione citata pag. 12.

(13) Nel decorso di questa memoria, cioè nella seconda parte, sarà meglio discussa la verisimiglianza di tali caratteri. 
ftata nello fqualo vivente or petrificato, chi non vede che quefto ittiolito non può in alcuna manicra riferirfi alla fpezic delli carcaria? ma che rintracciarti deve la fpezic fua in quella fuddivifione di fiuali, che d'appreffo gli altri comuni caratteri hanno quello di poffedere uno fpiraglio (14) per parte dietro degli occhj, alla veduta di cui il rempre inclito M. de la Cepèdc collocò lo fqualo (15) ruffetto.

5. XiV. Quefto chiarifimo profeffore rilevò cfiC 2

Atcte

(14) Ved. M. de la Cepède op. cit. Tom. I. pag. 22ro

(15) Nome comune al maschio ed alla femmina. Ved. M. de la Cepède opera cit. loco c. Incontrasi in Rondelezio ( I.ib. XIII. de piscibus cap. VII. pag. 330 . Lugdun. 1554) dato il nome di cirbicula Aristotelis, e nel vernacolo di Montpellier quello di charz allo squalo russetto, ed al cap. VIII. alla pdy. 333. del libro citato vedesi dato il nome di cabicula saxatilis, in lingua di Montpellier quello di catio rochiero ad altro squalo che non è diverso dal suddetto, se non che nella grandezza: grandezza che costituisce soltanto la diversità di sesso di una identica spezie. Aldrovandi per l'opposto della suddetta spezie forma un genere, che poi suddivide e distingue col nome di catulus major Salviani, e di catulus minor ejusdem. (Ved. Ulissis Aldrovandi de piscibus Lib. V. Bononiz 1639. Lib. III. cap. XXXIV. pag. 390. e 391.) Ora qual confusione non incontrasi nel rettificare li sinonimi dei pesci, che sì disparati nomi si meritarono!

Egli fu dinque per non aumentare la confusione ch' io usai nella nostra italica favella il nome di russetto dedot to dalla voce francese roussette, nominato nel veneto dialetto pesce gatta. In quanto poi all'aver io usato il nome di renardo invece di volpe, essendo questa estrinseca cosa, e non importante all'argomento, non ne farò alcuna parola. 
$20^{\circ}$

Atcre una intereffantifima proporzione fra la totale lunghezza di qualiafi carcaria (I 6 ) ed il contorno di un lato della relativa mafcella fuperiore, mifurato dall'angolo di congiunzione delle due mafeclle fuperiore ed inferiore fino all' eftremitì del lato della fuperiore mafcella medefima. Io mi fono molto ftudiato di rilcvare nel noftro ittiolito il punto di congituzione delle mafcelle, ma inutilmente, poichè neffun indizio di mafcelle vi comparifce, e li denti che vi fono confufamente diAtribuiti (17) moftrano effere fpoftati dal loro fito e difperfi in ferie oltre il conveniente allungata. Difpenfarmi pertanto non poffo dal dedurre, che nel noftro ittiolito le accennate mifure non fono applicabili per il confronto, nè fervir poffono di ccrto e ficuro appoggio per farne alcuna indue zione.

6. XV. Ad onta di ciò à gitoco forza l'andarmene più innanzi. Dietro mifure efattifime, e dictro computi (I8) di ogni eccezione maggiori rifulta, che il margine più lungo della parte fmaltata di uno delli più grandi denti di un giovane carcaria fta alla lunghezza totale del carcaria Ateffo come I al 200. Partendo da quefto dato irrefragabile

(i6) Ved. M. de la Cepède 1. c. pag. I76.

(17) Ved. Ia mia descrizione ec. pag. I2.

(18) Ved. MI. de la Cepède I. c. pag. 206. 
gabile pafar convicne a rintracciale nol petrificato fqualo un talc rapporto.

§. XVI. Offervati frattanto li denti maggiori di quefto pefee ingranditi del doppio con lenti, che un tal foco all' incirca poffeggono, fi potè vedere una differente tinta fopra $\mathrm{i}$ medefimi, clue in due porzioni concorre a diftingucrli. L'una porzione bianca lifcia e rifplendente riguarda il loro apice, l'altra di colore caftagn'ofcuro qualche cora afpra e ftriata fpetta alla divaricata loro bafe. Effi tali fono, quali delincati fi veggono alle figure II. III. IV. Quefti denti dal fito di rale biancheggiante porzione fino al loro apice (porzione che puoffi confiderare per il fuo rifplendimento, ed a veramente finaltata) poffeggono un lato tale, che non eccede in lunghezza le due lince (s millimetri). E poichè dalla fupputazione (19) qui inferiormente annotata rifulta, che la lunghezza dell' ittiolito forpaffa di molto la lingliczza che aver dovrebbe fe folfe un cane carcaria, così ne fcgue che dalla fiftemazione di una

tale

(19) La totale lunghezza dell' ittiolito si è di 1510. millimetri; il maggior lato della porzione smaltata di uno delli maggiori denti del medesimo si è di 5 millimetri, e però sta questo a quella come I a 302 , ma il lato analogo di uno delli maggiori denti di un giovane carcaria secondo le accennate misure sta come I al 200 , dunque il nostro sorpassa di 102 il termine ricercato per poter essere uno squalo carcaria, 
22

sale fpezie fi debba efcludere. E quantunque le dimenfioni delli denti del carcaria non aumentino di pari paffo, e nclla proporzione della lunghezza del loro corpo, ciò non pertanto non può accadere che il lato analogo di uno delli maggiori denti fi poffa ritrovare (20) affolutamente più breve, mentre la lunghezza dell' animale medefimo ii ritrova accrefciuta; il che arriverebbe fe nella rpezie delli carcaria includerfi dopeffe il noftro ittiolito .

§. XVII. Giova frattanto rimovere un obbietto che fento farmi : li denti dell' ittiolito potrebbero cfrer caduti nell' atto dello fpaccarfi del pefee, potrebbero quindi effere finarriti e cadere del tutto

per

(20) Se li denti delli carcaria, ed in conseguenza la porzione smaltata delli medesimi crescesse in proporzione sell' aumento in Iunghezza del loro corpo, la porzione smaltata di uno delli maggiori denti dell'ittiolito, se fosse un carcaria, aver dovrebbe il suo naggior lato lungo millimetri $7 \frac{12}{20}$; poichè la sua lunghezza totale è di millimetri 1510 , mentre il lato analogo della porzione smal. tata del carcaria mis'urato da M. de la Cepède si è millimetri 5 , e la sua totale lunghezza è di 1000 millimetri: ma l'aumento del centl non cresce in proporzione, dunque dev' esser meno di millimetri $7 \frac{11}{20}$; deve peraltro avere più di 5 millimetri, poichè se fosse eguale ai 5 millimetri niente nei denti crescerebbe, crescendo la lunghezza del corpo; e se fosse eguale ai 5 millimetri, come è nel nostro ittiolito, la lunghezza del dente rimasta sarebbe la stessa, cresciuta essendo la sua totale-lunghezza. La prima cosa è contraria alle osservazioni di M. de la Cepède, la seconda è assurda. 
per confeguenża l' accennato computo. A ciò rifpondo, che fe ftati vi folfero denti ancora più grandi degli offervabili in queft'archetipo, rimanervi arrebbe dovuto nella pietra Atefra delli medefimi qualche reftigio; ma nulla di ciò fcorgendoli, ne fegue che lungi dall'inferir qualche dubbiczza, ferve invece tutto quefto di appoggio per credere che il noftro ittiolito non abbia appartenenza colli carcaria predetti .

§. XVIII. Il fupporre poi che li denti maggiori di quefto pefee foffero caduti o prima, o contcmporancamente al fuo impetrincnto non fembra verifimile, perchè quand' anche fi voleffe crederlo un carcaria, non farebb'effo giunto a tale grandezza o a tal' età, per cui perdere aveffe potuto alcuni dei maggiori fuoi denti; oltre di che quefta fuppofizione mancanclo di ogni fondamento c di ogni offervazione di fatto, non fembra che apportar polfa veruna cecczione.

5. XIX. Se taluno fupporice roleffe che la giovanezza dello fqualo petrificato foffe la cagione, per cui comparire ancora non aveffe potuto la traccia delli denti conformati a guifa di $\mathrm{fega}_{\mathrm{a}}$, io mi farci a rifpondere in quefta maniera: ben di rado fi dà che gli efferi inticramente fviluppati portino diverfa conformazione di parti, bensi è noto che alcuni cfferi nelle diverfe loro età vengono rapprefentati di forme diverfe, perfin ad indur nell' c- 
quivoco di crederli di rpezie differente; ma nel cafo noftro rifcontro un fatto, ed ì che anche (21) i piccoli cani carcaria, e di minor dimenfione del noftro ittiolito, hanno li denti fatti a fega. Nel noftro archeripo effendofi offervati li denti con lenti famplici (non fidandofi della femplice oculare ifpezione) fi fono ritrovati mancanti (22) dei ricer-

cati

(21) Mi venne spedito da Venezia, il che si fu alla fine del passato giugno, un piccolo pesce lamia (squal. carcsria Linn.), la cui totale lunghezza non eccede gli undeci decimetri oltre ad un centimetro, eppure li suoi denti anche ad occhio mudo con molta precisione si veggono lateralmente conformati a guisa di sega .

Misurato il maggior lato della porzione smaltata di uno delli maggiori denti prominente fuori della polle, che legato lo tiene alla mascella, possede questo la lunghezza di 7 millimetri.

Misurato il lato della mascella superiore dall' angolo delle due mascelle sino alla sommiti del medesimo lato di detta superiore mascella, si è ritrovato avere la estensione di un decimetro piuttosto crescente. Dunque il lato di questa mascella sta alla totale lunghezza di questo squalo rilevata come sopra in i decimetri, ed un centimetro, come $I$ ad II all' incirca. Il che perfettamente quadra colle osservazioni di MI. de la Cepède sopra i carcaria. Ved. M. de la Cepède 1. c. Tom. I. pag. 176. Ad onta delle molte precauzioni usate, egli fu con mio dispiacere di non aver potuto preservare il corpo di questo piccolo lamia, il cui capo peraltro ben disseccato io conservo.

(22) Ved. le fig. II*. III*. IV*., il cui disegno venne eseguito dietro diligentissimo csame fatto con lenti semplici. A colpo d'occhio può quindi giudicare ogn' uno se sieno essi denti conformati a guisa di sega, o sc piuttosto i loro lati sieno levigatissimi ed incavati a foggia di guscio. 
cati dentelli nci loro margini laterali; non è dunqae la fua giovinezza la cagione della marginale levigatezza delli fuoi denti.

§. XX. Ma attenendofi ai fatti, il chiarifimo M. de la Cepède ha rilcvato che,, allora quando 10 fqualo carcaria (23) è giovaniffumo (trés-jeune) non moftra che un fol ordine di denci, nei quali fcorgefi, e folo qualche volta dei leggieri dentclli „. Egli dumque parla dei carcaria giovanifimi, cioè di quelli (interpteterei) che da. qualche pollice di lunghezza, o dalli duc decimetri (24) non cccedono li tre picdi, od al piì un metro (2 j).

$$
\mathrm{D}
$$$$
\text { Egli }
$$

(23) Ved. M. de la Cepède 1. c. pag. $177^{\circ}$

(24) Dopo aver parlato del carcaria giovanissimo M. de Ia Cepède, così si esprime, mais à mesure qu'il se développe, il en presente un plus grand nombre de rangées ", li quali in conseguenza delle cose dette di sopra sono denti addentellati. Inoltre il carcaria che il medesimo Autore rammentò, $c$ disse avere in Iunghezza rooo millimetri ( 37 pollici) facilmente fornito di denti serrati, non l' ha egli detto giovanissimo, ma soltanto giovane ló nominò. Dal che, se non erro, puossi dedurre, che li carcaria per dirsi giovanissimi debbono stare al di sotto, ed io creảo di molto, alli trc piedi di lunghezza. Dí fatto il carcaria pervenutomi ultimamente dall'Adriatico lungo I decimetri ed I centimetro, vedesi ormai dotato di cinque ordini di denti serrati .

(25) Nell' ittiologia veronese Parte II. pag. 10. tav. III. fig. 1 . viene rappresentato, e descritto un pesce lamia (sq. carcharias Linn.) Il benemerito Autore crede, o mostra credere, che esso squalo esser dovesse dotato di denti serrati, e ciò soltanto in supposizione, giacchè neppure un solo dente possede quello squalo. Di pili dietro la citazione della fig. II. tav. I., ove si contempla 
Egli ì in quefti carcaria aventi un fol ordine di denti, fe mal non mi appongo, che vedefi a mala pena, e qualche volta foltanto dei denti leggiermentc addentellati. Il rinomatilimo Autore non Soggiunge di qual lunghezza di corpo foffero li carcaria da clfo veduti coi denti come fopra: una dal contefto dell opera fua baftantemente rifulta, ch'efler doveficro (très-jeunes), cioc̀ giovanifimi (26) non eccedenti li tre picdi, od un metro in Junghezza .

Dictro tutto ciò io mi lufingo, che anche it fig. co: Gazola dotato di profonde cognizioni full' argुomento polfa incco convenire ne!l' efeludere que- . fto pefce dalla rpezic delli carcarin.

PAR-

delineato un bellissimo dente di pesce Iamia, il cui maggior lato compresa pur la radice si c̀ di 22 lince ( 5 centim.), così si esprime alla pag. XII.,, non ignorandosi ora, che questi corpi creduti un tcmpo lingue di animali impetrite sono veri denti lapidefatti del pesce sin qui descritto „,. Dunque anche il pesce colà descritto, quantunque minore di 7 decimetri in lunghezza dovrebbe avere a senso dell' crnatissimo Autore li denti serrati. Molto piu forte ragione sarebbevi per credere, (allorchè fosse un pesce lamia) aver dovesse scrrati li denti il nostro ittiolito, lungo essendo più di 15 decimetri.

(26) Tale spicgazione a parer mio pud scrvire altresi di schiarimento al compendioso passo di Linnco di Gmelin, dove dice, che il pesce lamia (squalus carcharias) si trova, sex, juniori paucioribus, dentium, frequenter fossilium, triangularium, juniori nondum serratorum, ordinibus horrens. Vedete Tom. I. Parte III. pag. 1499. Lond. cdit. XIII. 


\section{I) A R T E II.}

§. XXI. Ora egli ì mestieri fare ogni sforzo per cercal di conofecre più da vicino che lia poffibile il noftro ittiolito. Per ciò efeguire lafciando intatto il conturno del pefee medelimo alla fig. I. delineato in grandezza dodici volte minore del naturale, fonoli prefe in difegno le di lui parti calatteriftiche, rettificandole a parte a parte fopra di appolita fala, orizzontalmente lituandole, lafiato avendo nel tempo fteffo alle varic fue parti - la maggiore poffibile identita, tali in fomma, quali alla figura $V$. delineate ii veggono. Retta folo a notare, che nella coftruzione di detta V. figura le linec tracciatc da' punti s'impiegarono per indicare le parti del pefce mancanti. Quali parti fegnate per approfimazione fopra le accidentalità ch' egli prefenta, fervono a far trafpirare per entro alle di lui involute da tenebre antichiflime fpoglie ciò che al prino fuo afpetto par che fi afconda.

§. XXII. Per non ripetere qui la fpicgazione di esse tavole, e nemmeno il rifultato del quadro delle fue dimenfioni, or mi produco ficcome feci al §. III. della citata mia lettera, alla fiftemzzione del genere, a cui fembra cffere da riferirfi quefto petrificato pefece

Bonatcre nella Enciclopedia definife il genere 
28

Squalus (2i) con quefte parole: " le corps oblong, comprimé par les côtés, oli arondi, \& coulvert d'une peau chagrinée „. Il nottro animale ha il corpo luingo, la pelle zigrinata; la fua rotonditì fi $\mathrm{fa}$, e fi vede che dalla compreffione viene altcrata, ma la traccia ve l'indica, perciò fembra non equivocarfi nel definitlo per un canc marino: Squalus (28) Linnai f. n. 397.

§. XXIII. Paffiamo a rintracciarne la fpczic. Si prenda la molfa partendo dalla conliderazione del capo del pefce medelimo. Ed in quanto al fuo mufo, fino dalla prima parte di quefta mcmoria ho indicato, che la volta offea dicl palato fuo, come arvenire doveva, appianata fi trova. Lo Atcfo è forza accordarc in riguardo al fornice del flo cranio, pcl cui (22) liacaffare, nonchè pcr il divaricato allargamento delle parti molli. fpezialmente labiali effer pottcbbe arrivato, che if mufo

(27) Ved. Tableau enciclop, ec. icthyol. pag. 6.

(28) Ved. Linn. s, n. edit. XII. Vindobon.

(29) Puossi di ciò render qualche ragione, supponendo che le sutture longitudinali del cranio molto facili a ssgrcgarsi fra loro nei pesci, siensi in questo realmente separate; e che restando fisso, come a punto di appoggia il suo 11250 , siensi mosse in giro le ossa parietali e temporali, col determinare in tal guisa il corpo del pesce a prendere una figura circolare o quasi circolare; ad cssa forma portato vie maggiormente dalle parti molli dello stesso. Con ciò resterebbe anatomicamente spiegato come il muso cuneiforme di un volpina di mare, esser potrebbe tale, quale si è quello de! nostro ittiolito. 
mulo cunciforme di uno fqualo volpe; divenuto foffe di rotondiforme figura.

§. XXIV. La macchia od impronta marcata $x_{0}$ fig. I. ¿̀ diftinta non folo da una cute più fottilmẹte zigrinata, la quale non fenza però qualche irregolarità la circofcrive, ma pur anco da un leggierifimo rifaltino ó rilievo, da cui viene conterminata nel fuo conturno. Ora la fituazione di quefta inacchia molto anteriore, la fua ellittica figura le fue dimentioni di grandezza efcludendolo dalla fomiglianza del forame uditorio che in tali animali pur efifte, la fanno credere effere piuttofto ftata quefta la regione dell' occhio ricoperto quafi per intiero dalla fua inembrana, corne quella che portano alcuni degli fquali viventi.

§. XXV. La lettera z. ferve a marcare nel capo fteffo la macchia, od impronta tracciata da varie grinze che $s$ interfecano fopra una cute coriacea, la qual effendo nella pietra medefima q̨ualche cofa depreffa, lafcia luogo nel tempo fteffo a dubitare re poffa effere uno degli fpiragli, o fe meglio per la fua fituazione effere poffa formata dalle aperturc branchiali fra fefteffe nel morire dell'animale aggruppate .

§. XXVI. Denti acuti all' apice dilatati alla bafe, quattro linee allungati all' incirca, circolarmente difpofti in tre ordini confufamente diftribuiti rono quelli di quefto folfile fqualo. Gli fteffi denti 
30

spezialmente li maggiori motrano poco al difopra della metì di uno o dell'altro dei loro lati una fpezic d'incifura o di ricntramento, ed inoltre ai loro margini da ciafcun lato una tenue refta tagliente, che dal corpo dei denti fteli partendo viene ad elfere fcavata a guifa di gufcio. Nè acquictarc volcndomi all' ifpezione dell' occhio, all' olfervazione delle acconnate lenti divenni, per il cui mezzo (30) confrimare potei, quanto aveva ad occhio nudo veduto.

Qucfa forma di denti che con tutta chisrezza fi riconofce, limita queft' ittiolito alle feguenti fpezic, c fono lo Squalus gluzcus, to Squalus indiius, lo fqualus denticulatus, lo fqualus vulpes.

§. XXVII. Non può effere poi lo fqualus glaucus, perchè quefto ha la fua pinna candisle conformata (31) a guifa di luna crefcente, ed ha le fuc pinne petturali molto allungate a differenza del noftro ittiolito .

Non può efferc lo fqualus indicus, perchè oltre all'altre particolariti rimarcabili nel di lui corpo; la di lui coda fpezialmente (32) ¿̀ uniloba, men-: tre biloba è la colla dell' ittiolito.

Non

(30) Ved. la fig. II*. III*. IV*. non che la citata de. scrizione alla pag. 12 .

(31) Ved. M. de la Cepède, op. cit. loc. cit. pag. 213. Pl. 3 .

(32) Ved, MI. de la Ciepède, op. l. c. pag, 280. 
Non può effere lo Squalus denticulatus, perchè febbene la pinna caudale di quelto fqualo lia divifa in due lobi, rimarcafi che il lobo inferiore della fua coda ha origine all' eftremitì della coda medefima. Al contrario la divifione del lobo inferiore della coda dell'ittiolito comincia a flai piì al difopra. Inoltre il lobo inferiore della coda dell'ittiolito è di gran lunga più b:cre del fuperiore, mentic quello dello Squalus denticulatus è più grande del fuperiore, ed è cone fuddivifo (33) in tre picculi lobi.

Refta dunjue a credere ch'egli aver poffa qualche appartencuza $(; 4)$ collo Squalus sulpes.

9. XXVIII. Bafterebbe quanto fi è tcitè accennato per crederlo a tale fpezic efelufivanente appartenente; ma ragion ruole che fi efaminino partitamente gli altri fuoi caratteri.

La cute fua qua c là cofperfa di rarj centri, gli uni ridondanti di piccoli punti tuberculari, gli altri di lif́cio afpetto piuttofto che di fquame forniti, giuftifica quanto (35) altra volta fi diffe, cios̀ che la fua pclle è piuttofo lifcia e zigrinata, di quello clie ricoperta di foguame.

(33) Ved. M. de la Cepède 1. c.

(34) Ved. s. n. Linn. Cur. J. F. Gmelin, edit. XIII. Londin. I7\$9. Tom. 1. Part. III. pag. 1496.

(35) Ved, la citata descrizione pag. I 9. 
;2

Veggafi ora $r e$ un tale carattere al confronto regga con quello della volpe marina. Gefnero afficura che la cute di tale rpezie di pefce e per la forma, e per la coftituzione fua $(36)$ a quella corrifponde degli fquali zigrinati. La Enciclopedia parla di un renardo o fqualo volpino, il quale coperto crà di (37) lifcia pelle: cıò è ben diverfo dall' aver avuto una cute fquamofa. Ma fentire conviene la voce come di giudice del chiarifimo M. de la Cepède. Egli afferife, che la pelle del renardo di mare è ricoperta di piccolifjime tuberofita (38) o fcaglie. Quefte due proprictì feparatamente prefe, effento fra loro diverle, richieggono effere complefivanente confiderate. Egli, o lignor profeffore, conofec l'intrinfeco fenfo della propolizione alla ricordanza richiamando, ciocchè fu da lui offervato, e lo fu pure da me fopra la pclle dello fqualo volpe dell'Adriatico (32), veduto in Chiozza preffo l'egregio fig. ab. Chiereghin, allorchè mi conceffe cffo feguirlo nclla efeguitavi fortunatifima corfa. Quel volpino moftrava la cuite af-

(3б) Ved. op. cit. 1. c. pag. 1405 . dove parla del marino canc volpe, e dice, che esso porta cutem ad galeos arcedentem, sed inagis levem.

(37) Ved. Encyclopéd. méthod. hist. nat. des animaux Tom. III. pag. 201. Padoute 1787 .

(38) Ved. MT. de la Cepède 1. c. pag. 268.

(39) Anche il mare Adriatico è patria dello squalo volpe. 
arpriflima, fe fi toccava colla mano fcorrente dall' eftremitì della coda verfo il fuo capo, ed al contrario la fua afprezza non fi percepiva, fe fdrucciolare la inano facevafi dal capo verfo la coda. Del qual fenomeno rintracciandone la cagione, non d'altro dipenderc fi conobbe, fuorchè dall' inferzione dei picciolifrini fuoi tubercoli: inferzione fatta nella maniera Ateffa embricata delle fquame degli altri pefci. Quindi s'intende come il zigrino della fua cute paragonabile fia a fpoglie fquamofe. Efattiffima dunque fi è la efpreffione di $M$. de la Cepède, che diffe di tale natura dotato lo fqualo volpe.

§. XXIX. Non diverfamente topo è intendere ia deferizione ittiologica (40) di Bonaterre. Bafta una volta fola l'aver veduto il renardo, o voglian dirlo volpino di mare, qual cgli fi è, per efferne pienamente convinti. Del refto fe taluno crvi che un folo veduto ne abbia, la cui pelle anche in parte, o per inticro coperta folfe di fcaglic, ficcume non avià per mia parte ad effer difdetto di ciò che ha offervato, così mi lufingo che altri negar non faprì di averio noi veduto zigrinato.

\$. XXX. Quefto ittiolito che tanto combina colla cute dello fqualo volpe, fembra combinare altresi

$$
\text { E nella }
$$

(40) Ved. Tableau encyclop, \&cc. iđhyol. pag. S. 
34

nella cllittica forma del fuo corpo. Se altro non foffe, li frammenti della propria foftanza, che fotto il fuo addome fparfi ritrovanti, fanno baftevole teftimonianza della interrotta fua continuità; ficcome pure la corrofione del fuo dorfo nel lito inarcato op fig. I. e fig. V. puofi defumere da fimil cagione. Ciò non pertanto aftrazione facendo dagli accidentali aumenti e decrementi da proporzioni non moftra egli quefto petrificato fqualo $(41)$ la forma di corpo del tutto ellittica?

§. XXXI. La intertizione di sì gran tratto dell' addominale contorno nell'ittiolito feco ravroglie la perdita delle fue pinne ventrali ed anali.

§. XXXII. Le due pinne pettorali e per i loro contorni, e per la loro forma fono affatto limili a quelle dells volpi di Rondelczio, di Gefnero ce. le cui figure confultare fi poffono negli originali frrittori .

§. XXXII. L' antcriore pinna dorfale manca ncll'ittiolito, ma nel luogo marcato $m$ fig. I. che corrifponde all'incirca al mezzo del dorfo fi feopre qualche (42) rudimento di effa pinna.

\section{§. XXXIV.}

(41) Facendo qualche riflesso agli sparsi frammenti addominali, sembra che il limite del suo addome possa verisimilmente essere stato non molto lontano dalla linea tracciata da' punti e marcata $t s r$. Ved. fig. V.

(42) Ved. Linn. 1. cit. pag. I 496. dove si legge così, Vulpes. . . pinna dorsalis prior in medio dorsi. 
§. XXXIV. La pinna dorfale pofteriore rotta nello fpaccarfi del perce, venne inneftata nel fito in cui mirafi; rimane però il dubbio della convenevole fua adatiazione.

§. XXXV. Relta a parlat della coda - Nei pefei c̀ quefto J'organo il pit̀ mobile ed il più fleflibile, in grazia di cui rapidifime vengono efeguite le loro marcie, e cui ineres̀ gli sforzi piu violenti praticati vengono, onde procacciarfi la fuga dai naturali, o artifizioli perigli . Di tale veriti convinto vengo all'applicazione: ma fi efamini prima la immaginata coftuzione della figura V.

§. XXXVI. Lo fqualo della figura V. porta la coda conformata come quella della figura I. per il lungo tratto della porzione Q. E.F., fuorchè la direzion fua ¿ qualche cofa piì orizzontale. Nel punto Q. effervi dovrebbe la foffetta (43) triangolare, ma è pofibile che fiafi perduta nella fua petrificazione. Ciò che meritar fembra qualche rifleffo fi è la poca fua larghezza nel luogo di fua origine di già marcato $Q$. non eccedente nel fuo perpendicolare diametro certamente li 4 pollici ( $I$. decimetro ed $\&$ millimetri); il che beniffimo fi accorda cogli originali viventi di quella spezie che

$$
\text { E } 2
$$
più

(43) Ved. MI. de la Cepède, op. cit. 1. c, pag. 268. 
36

più fopra fi è nominata. Diftante di qui per tin tratto di 4 pollici od 1 linca (piì di 1 t contimetri) ritrovafi la biloba fua divifione.

Al di lì dell'inferzione dell'inferiore lobo calldale vedefi nella figura I. ombreggiato il groffo tronco del lobo fuperiore un po' piit in largo di quello fia il tratto ftelfo di colla nella fig. V. Ma ciù fi fece appoftatamente, perchè attenzione facendo ropra l'archetipo nel luogo corrifpondente, effcrvi fi diftingue in parte una fpezic di sfacimento, ed in parte una fpczie di trapelazione della foftanza forfe la piì tenue del pefec infinuatafi per entro alla pietra per la compreflione. facilmentc operatali o contemporaneamente, o pofteriormente al fuo impetrimento. Sicchè il confine. del vero contorno inferiure di detto tratto di lobo. deila eoda non. fenza ragione. gindicare fi può limitato ncll'originale a quella linca che nclla fig. I. predetta puofi mirare piì profondamente marcata dell' altre che le fono vicine, ed è diftinta colle letterc $\vartheta$ \%

Inoltre, poichè il pefce a chiara luce ora efposto fi trova, egli c̀ nel fito che corrifponde al!s fezione EF. fig. V. e fig. I., in cui vedefi all'efrema porzione di coda $G g f$. pel tratto di piu di una linea e mezzo ( 4 millimetri) produrfi al difotto una porzioncella di cute marcata $\alpha \beta \omega$ fig. I. per tale giudicata si per la cquabile fua giaci- 
tula; si pel fuo zigrinamento deI tutto fimile a quello del groffo tronco caudale Q E.F.

Affinchi la coda di tale archetipo crederfi poreffe continuata, converrebbe che le fue vertebre le une dietio le alire ii fuffeguiffero con decremento quafi infenfibile. Ma per il contratio ritovafi che l'ultima rifibile vetebra in EF. fig. I. ha una larghezza triangolare di quafi 5 lince (un centimetro crefeente), mentre la prima vertebra in $g$. della caudale eftremità $g f$. ha la trafverfale fua larghezza minore di una linca c mezzo ( 3 , millimetri circa). Di più quefta piccoliffima vel' tcbra a differenza: della prima or nominata è ricoperta di cute, con che maggiormente compiendefi- la sproporzionata convenicnza, la impoffibile continuità di quefte due porzioni, e la mancanza di un intermedio tratto di coda.

6. XXXVII. Si ftacchi pertanto fig. I. la calts dalc eftremità $G g f \cdot c$ rrogliendola dal difopra in giì, qual dovrebb'cffere, la fi porti dal fito EF. tanto diftante, quanto importi un congruo graduato decremento delle fue vertebre, c fi vedrà cffa fig. V. pofta in $g G f$. di manicra che comparirà il lungo tratto di coda EG. F g., il che per lo appunto ¿े, quanto puofli fupporre mancante nella medefima, tracciate cffendo così le fue vertebie in continuata e ben conneffa degradazione.

§. XXXVIII. La predetta interporta porzione di coda 


\section{8}

coda tracciata con feric di punti fig. V., che gli sliftenti vilibili tronchi già riunife, c leggiermente incurvata, prende una forma del tutto fimile a quella deile volpi marine, cguagliandofi all' incirca al rimanente del fuo corpo. Di fatto il difegno fteffo coftituifce un tutto eftefo a 6 piedi, 3 pollici, ed $\$$ lince ( 2 metri, 4 centimetri, e 6 millimetri) mifure peraltro applicabili alle varic lievi curvature tutt'ora cfiftenti nel difegno predetto, ch' è piaciuto di così elibire per ricordarc poffibilmente le rembianze dell'ittiolito medefimo. Ma la coda di uno fqualo conformata a guifa di falce, lunga o quafi lunga quanto il rimanente del corpo (44), c che lo fupera (45), compete allo fqualo volpe, denque egli è poffibile che it noftro itriolito tal cffer poffa.

§. XXXIX. Sopfa la coftruzione della citata figura $V$. replicare taluno potrcbbe: veggo beniffimo che l'apice della coda $g f$. corrifponder non può per continuità al groffo tronco della coda in

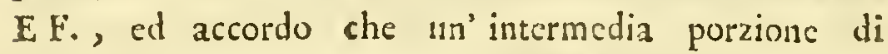
coda vi manchi, ma quefta dove ì ? come fi ct fmarrita? Ciù puoffi fpiegare fupponendo il perce efferc

(44) Ved. Tableau encyclop. \& méthod. \&c. idhyol. pag. 8 .

(45) Ved. Artedi Petri aquatilium animalium historiæ Lib. I. Romx 1554, synonym. 96 . 
effere thato colto, mentre ripiegata e guizzante teneva (46) la coda, di cui ora una interpolta porzione della medefima venga a rimanere latente.

§. XL. Chiamo latente detta porzione di coda, non perchè io creda ch'effa realmente fi appiari per entro alle lamine della pietra medefima, ina perchè non è certa la foppreffione fua tale quale (i è pofta, parlando intorno alla poffibiliti di tale fpezic. Io la dico dunque latente fotto tal vifta, perchè li fottrac alle prefenti ricerche. E' però vero che del fuppofto tortuofo fuo giro fpiegare potrebbefi la foppreffione o anteriormente, o contemporancamente al di lui impetrimento o per lacerazione, o per qualunque altro avvenimento, o perchè dai poco oculati minatori infranto effendofi l'incontro fuo, fmarrita fi folfe di detta polzione ogni traccia.

5. XLI. Deggio poi aggiungere a quefto propolito alcuni argomenti, dai quali puoffi inferire - la pluralitì (47) delle spezic, o la degradazion (48) della rpezie degli fquali volpini.

Se agli feritti dell' antico Salviani preftar fede fi deve (49), gindicar egli è forza, che la volpe

ma-

(46) Ved. sopra 6. XXXV.

(47) Ved. M. de la Cepède op. cit. Tom. II. Discours sur la durée des especes pag. XXXIV.

(48) Ved. 1. c. pag. XXV.

(49) Ved. Aquatilium animalium historice Lib. I. cum 
marina da effo defcritta di fpezie fia realmonte diverfa da quella di Rondelezio. Il più volte menzionato Uliffe Aldrovandi diede notizia di una fpezie (5o) di fqualo, che per la furma della fua coda alla fpezic delle volpi di mare fu attribuito.

corum formis are excussis: Hippol. Salviano Typhenale Audore homx 1554. p. I34. D. Historia quadragesima de vulpecula, di cui la figura delineata si vede alla pag. 130 colla iscrizione latina rulpecula, a canto cui leggesi il nome italiano pesce sorcie: il quale ha la coda ristrettissima, lunga non quanto però il suo corpo, ed uniloba: inoltre ha il rostro assai più lungo di quello della vulpecula di Pondelczio. Tali differenze nei detti pesci incontrate indussero l'Aldrovandi, come si può vedere alla pag. 396 della cit. sua op., ad esprimersi così : An ne fortassis duo sunt genera? Sebbene a vero dire li generi suoi costituiscano appo li più moderni ittiologi delle spezie soltanto.

(5o) La figura di detto squalo vedesi riportata alla pag. 397 dell opera sua, sopra il cui vertice scritto si legge vulpecula alia. Inclina dunque egli medesimo questo Autore a crederlo appartenente ai volpini, ma però dagli stessi specificamente differente. Di fatto dalle misure prese delle proporzioni della citata figura risulta, ch' essa coda sta alla totale lunghezza residua del pesce come I a $2 \frac{112}{167}$; essa è dunque una metà e $\frac{112}{167}$, più breve di ciò ch' esser dovrebbe per eguagliare almeno quella dei cogniti squali volpi. Rimane pertanto luogo a credersi questa pure o una degradazione di spezie, ovvero una spezie a quella prossimamente vicina. E ciò tanto più, quanto che il rostro di questo è molto più acuminato, ed orrido di quello degli altri comunemente cogniti squali volpini. Eccone le proporzioni tratte dall' cnunziata figura.

La coda in lunghezza decim. 1 , centim. 6, millim. 7.

La testa ed il corpo assieme presi sono lung. decim. 4, centim. 4, millim. 6 .

Somma totale decim, 6, cent, I, mill, is 
Altro efempio incontrafi di notabiliffma differenza in un volpino riportato da M. Daubenton; il racconto è prefo da Linnco, il quale ( 51 ) per la mafima parte ad una defcrizione riportoff di Willugh by .

§. XLII. Ma che vo io annoverando cotanto antiche offervazioni? non fi vede forfe ogni giomo paffare fott'occhio alcuno di confimili efempj nel regno animale? La fucceflione, il riproducimento, c la durata delle (52) fpezie fono innegabili, le quali ben lungi dall' efere caratterizzate per nuvftruofi producimenti ( 53 ), altro non coftituifcono che delie $(s ;)$ gradazioni dall' una all'altra.

(5I) Ved. Encyclop. méthod. \&xc, Tom. III. pag. 201. Padone 1787 . dove rilevasi, che il volpino preso I'anno r6or. nell' Oceano settentrionale era fornito di un solo foro da ciascheduna parte posteriormente agli occhi; era peraltro esso foro molto largo, e serviva ad uso di branchic ; per questo foro guardando scorgeasi esistere interiormeute quattro divisioni branchiali. Mancava egli dunque dei laterali, 'ed inferiori cinque pertugj branchiali attribuiti dagli Autori comunemente alle volpi di mare.

(52) Ved. M. di Buffon Histoire naturelle générale \& particulière, avec la description du cabinct du Roy. Second édition à Paris. De limpriméric royal 1750. T. II. des animaux Chap. 1. pag. 3. \& 10.

(53) Chiamasi mustro ogrii produzione orgarica, della quale o la conformazione, od il collocamento, ovvero il numero di alcune delle sue parti le ordinarie leggi non segue. Ved. Bonnet ouvres d'Histoire naturelle, \& de philosophie à Neuchatel 1779. Tom. V. Considérations sur les corps organiq. S. XXX. Des monstres pag. I02.

(54) Ved. M. de la Cepède op. cit. T. II. pag. XXXX. loc, cit. 
42

9. XLIII. E per vero dire quanto non è piì da filofolo to ammettere la ordinata fucceffione di quello che il paffaggio dall'una all'altra spezie difparatamente diverfa? Efifte in natura una non interrotta continuazione di anelli che negat non fi può, dacchè il padre di queft'augufta continuiti, roglio dire il Bonnet, ai filici ne diede moltiplicate e cosi grandi riprove. Sembra dunque ragionevole il cledere, che o una gradazione, o che più di una fpezic diafi di fquali volpini.

§. XLIV. Ma in quanto allo fqualo petrificato formerì egli una fpezic fua propria ? Tale queftione effere non fembra adequatamente folubile a cagione degli addotti argumenti, che militano in favore per una parte, e inilitano contro per l'altra. Certamente la dubbiczza prenderì forza maggiore ogni fiata che rifletrere vogliafi alla deformità della fua tefta, alla laccrazione del corpo; nonchè alla mancanza e frattura di alcune fuc pinne, finalmente all'ambiguità di fua coda, come difopra evidentemente fi cे dimoltrato.

\$. XLV. Che fe neppure all' anzidette poteffe appartencre, altro non refterebbe che confiderarlo come una fpezic novella, o riporlo in alcuna di quelle che fimilmente all' accaduto (is) ad altri petrificati

fi

(55) Ved. MI. de la Cepède op. cit. I. c. pag. LVII. 
fí rifcontran perdute. Che fe ciò foffe, cofa quindi ne feguirebbe? Non d'avvantaggio certifimamente; fe non che alla nuova fpezie darli dovrebbe una denominazione nuvella. $\mathrm{Ma}$ il nurovo nome derom gherebbe agl' innegabili rapporti dei fuoi caratteri con quelli del vero marino renardo o pefce volpe? ed alla profimiti di fus fpezic, che collocargli farebbe mefticri fubitamente vicina? In forza ilunque di portentofe accidentalıtà fembra che non fieno precifi e riconofcibili i caratteri, onde con licurezza detcrminare la fpezie di queftu fqualo.

\$. XLVI. In quanto poi alla lua patria, cone venendo ch'egli fia una delle fpezic mentovate $c$ cognite, particolarmente te tha lo fqualo volpe che troviam pure nell'Adriatico, fono cognite alcune delle località dove quefte abitano, ed abiteranno forfe in tali altre localita, che.finora non fono a cognizione. Se poi è quefta una fpezic nuova, chi può indovinare qual lia la patria fua? non $\mathrm{fa}_{\mathrm{a}}$ rebbe che un azzardare imnaginofamente il pronunziare fopra il fuo loco nativo.

§. XLVII. Mi rendo follecito troncar il tedia di quefta mia narrazione, il cui feopo fi riftrinfe a far conofece $I^{\circ}$. la impoffibilitì che quefto ittiolito fia uno fqualo carcaria, o pefee lamia. $2^{\circ}$. La poffibiliti che fia uno fqualo renardo, o pefce volpe, non cfelufa la poffibilitì di appartemere ad altra diftinta fpezic di volpini, oppure ad 
44

una nuora fpezic di fquali, fperie peraltro che per la confentaneiti delli caratteri fituarc dovrebbero li piì moderni metodifi proflimamente vicina a quella delle volpi marine, ficcome nella compendiofa mia defcrizione fino d'allora ho creduto pote: avanzaic.

Se voi, dottiffumo amico, che nel centro v'aggirate delle feienze tutte floridifimamente coltivate; fe nei ftupendi gabincti che in codefta inctropoli fervono d'iltruzione agli amatori delle naturali curiofità, trovate oggetti dai quali irtefragabili lumi. ritrarre ii poffano nel prefentc argomento, aficuratevi, celcbre fig. profeffore, che nell' eflerne potto a parte uni riufciranno gradirifimi. Ripoferò in feno al riputatifimo vofto giudizio, fe pronunziare vi piaccia con maggiore precilione di quel chi io non feci, la vera fpezie del cane marino petrificato, che $f u$ il foggetto di quento mio abbozzo; copra il quale io mi propongo di nulla più ag* giungcre, occupato ritrovandomi in altre frie applicazioni. Che fe nel propofito io $\mathrm{mi}$ folli accostato ai luninofi penfamenti voftri, quefta combinazione formerebbe per me il più grato compenfo. Frattanto paffo all'onore di afficurarvi della vera mia ftima c perfetta cunfiderazione.

Lonigo li 20. Gennaro $1 \mathrm{SO}_{7}$.

Francefco Orazio Scortigagna:

QuA 
Quadro delle (*) Dinensioni Dell'ittiolito.

Lunghezza prefa dall'una all'altra met. dec, cent, mill. catremità feguendo la vilibile infleffione della fua fpina. . . I. S. I.

Maggiore larghezza diamctrale della tefta

Lunghezza diametrale della tefta. 2. 4. 3 ;

Lunghezza del dorfo prefa. feguendo la inflefione fpinale . . . . 7. \$。

Lunghezza della coda prefa dal fito dell' apparente fuo incominciamento fino al fuo apice feguendo la fuperfiziale vilibile fut inflefione. . . . 4. S. it

Suo maggior dianetro dalla fominitì della fchiena all' apparente lembo dell' addome . . . . . . 3. is

rarghezza della coda nel fito dell' apparente fua origine. . . . . 4. 8.

Lato maggiore dell'appendicetta caudale lungo. . . . . , . . 2. 7 :

Lubo

(*) Spiegazione delle suddette misure.

Il metro equivale in lunghezza a trentasette pollici del piede reale di Parigi.

Il decimetro è la decima parte del metro.

Il centimetro è la centesima parte del metro.

Il millimetro è la millesima parte del metro. 
46

Lobo inferiore caudale mifurato dal meto dec. cent, millo fito della fua piì alta inferzione fino al fuo apice. . . . . . 1. 4. 9.

Pinna pettorale cfiftente in fito, lunga . . . . . . . . .

Altra pinna pettorale inueftata dall' artefice: cfifteva petrificata lungi dal capo dell' ittiolito per altrettanto intcrvallo di quello è lunga effa mede-

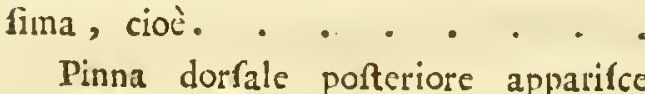
lunga . . . . . . . . .

Diftanza della fiddetta dalle apparenti traccie dell' anteriore pinna dorfale. - . * . . -
Lunghezza della macchia, od impronta anteriore polta. quafi all'eftemitì del mufo dell' ittiolito . . .

Larghezza della fuddetta . . .

I. 3. 7 .

Lunghezza della macchia, od impronta fuperiore e pofteriore del capo tterfo - . . . . . . . .

Larghezza della fuddetta . .

Diftanza da una all altra delle nominate due macchie . . . .

Lunghezza del maggior lato di uno delli inaggiori denti dell' ittiolito comprefa par anco la fua radice. . .

1. 2. 7 .

3. 6 .
3. 3

2.

2. $\%$

1. 6 .

1. 4. 8 . 9. 
Il maggior lato della porzione li-

Scia $\mathrm{c}$ biancheggiante di uno dclli maggiori denti dell'ittiolito non sccedente li . . . . . . .

\section{SIIEGAZIONE DELIE FIGURE.}

Rapprefenta la figura I. il difegno dell' ittiolito marcato nei fuoi contorni ranto dell' cfteriori cospicue fue parti, quanco delle interiori, delineato dodici volte ininore del naturale, fopra cui è da notarfi

A B C. Effere 1o fpazio occupato da triplice ferie di denti confufamente diftributiti, rilevati efiftere al num. di cento e tre, polti fuori di fcde. $a b c$. Officini del palato vifibile nell'ittiolito.

cd. Vertebre che penetrano per entro il capo. de. Vertebre dorfali.

egf. Vertebre della coda, in cui rimirali il tratto $\dot{G} g f$ dell' cftremità caudale fovrapporto al groffo tronco $\mathrm{EF}$. ed allo fpazio $\alpha \beta \omega$.

b. Appendicetta del lobo fuperiore caudale rivoltata all'infù contro l'ordine offervato in confimili fpezie di pefci.

i. Lobo inferiorc caudale.

l. Pinna dorfale pofteriore.

m. Rudimenti della pinna dorfale anteriore.

31. Pinna petrorale anteriorc. 
48

N. Pinna inneftata in quelta fituazione, fu rinvenuta petrificata nello ftrato medefimo, in cui fu prefo il pefee, dirtante dal mufo di effo per tanto intervallo, quanco all' incirca i lumga efla medeo fima .

op. Porzione di dorfo mancante nell' ittiolito.

Q. Difcefa clie dà origine alla fua coła.

rst. Situazione ove ii veggono traccie de!l'interrotta continuitì 'dell' addome.

$u v$. Porzione addorninale rimarcabile per ciò che verrì da offervarfi nella fig. V.

$x$. Macchia od impronta di figura ellittica dim fccrnibile nella pictra tanto per la qualitì dell'integumento, quanto a cagione di un rifaltino leggicrmente sevato fop:a il livello della pictra circonvicina .

z. Macchia od impronta di figura ellirtica, qualche cofa più depreffa della pietra che la circofcive, fegnata pur anco da grinze variamente interfecantili .

$\alpha \beta \omega$. Spazio di cute fottopofto all' eftremitio caudale $G g f$.

$\checkmark$ 6. Linea che indica il piì naturale confine inferiore del fuperior lobu caudale dell'ittiolito .

La II. figura efibifce il ritratto alla naturalc grandezza di uno delli maggiori denti dell'ittiolito, nel quale fi confidera in

y. La fua incifura in 
8. Il fuo apice actito che dì origine alla fpina bilatcrale $\delta \varepsilon . \delta \gamma\}$ in

$\delta \eta$. Il lato maggiore della porzione albeggiante c lifria di detto dente in

$\gamma$ \ท\&. La bafe o radice di detto dente di colore caftagn' ofcuro qualche poco afpra e ftriata.

La II*. figura rapprefenta il dente medefimo al 'eoppio circa ingrandito con lenti.

Le figurc III. c IV. indicano altri maggiori 'denti dell' ittiolito fleffo; avendo efpreffamente voluto far rimarcare nclla figura IV. li due denti, l'uno all'alow fonrapponte rnme fi offerva nell' ittiolito.

Lc figurc III ${ }^{\star}$. e IV ${ }^{\star}$. moltrano li denti predetti ragranditi all'incirca del doppio con Ienti.

La figura V. efprime il difegno dell' ittiolito siclla fuppofizione ch' cffo fia uno fqualo renardo, offia volpino; intorno il quale cade in acconcio notarfi che li contorni delle varie fue parti furono tradotti, ed hanno relazione tanto per la forma, quanto per la grandezza con quelli deila fig. I., e che li contorni tracciati con ferie di punti fervono a montrare le parti giudicate perdute ed appartenenti al pefee medefimo. Si ommifero in quefta figura le lettere fuperflue, e fi ritenncro quelle per il cui mezzo rilevare fi può a colpo d'occhio il loro fignificato, poichc̀ alle parti limili fi fono appontc le identiche lettere della fig. $\mathbf{I}$. 
5o

Refta folo a notare; che

op. Indica un breve tratto di dorfo lacerato nell' ittiolito .

$r s t$. Fa conofcere la-fituazione, in cui fi mis rano traccic della interrotta continuitì dell' addome a cui fi fegnò con ferie di punti il fuo limite giudicato il più convencyole a detta fpezie.

$u v$. Intervallo addominale in quefta figura ald lungato quafi duc volte più che nella figura $I . ;$ il che neceffariamente dovette feguire avendo roluto dare alla figura fteffa orizzontale pofizionc.

Q. Difcefa del dorfo dell'ittiolito. la quale dà origine al tronco della fua coda, le cui vertebre conuinciano in e. terminano in $f$.

$F g$. $E$ G. Porzione aggiunta di coda; in cua: rimarcafi una graduata decrefeente fcrie di vertebre giudicata la più conveniente al noftro arehes. tipo, ridurlo volendo all' integrità dell' indicata spczic.

$G g f$. Porzione di coda ftaccata dal luogo di fila accidentale e preternaturale pofizione trasferita ad una congrua diftanza, la quale coll' appendicetta fua $h$. comparifce rivoltata all' ingiì come negli originali della furriferita 1pezie.

F I N E. 

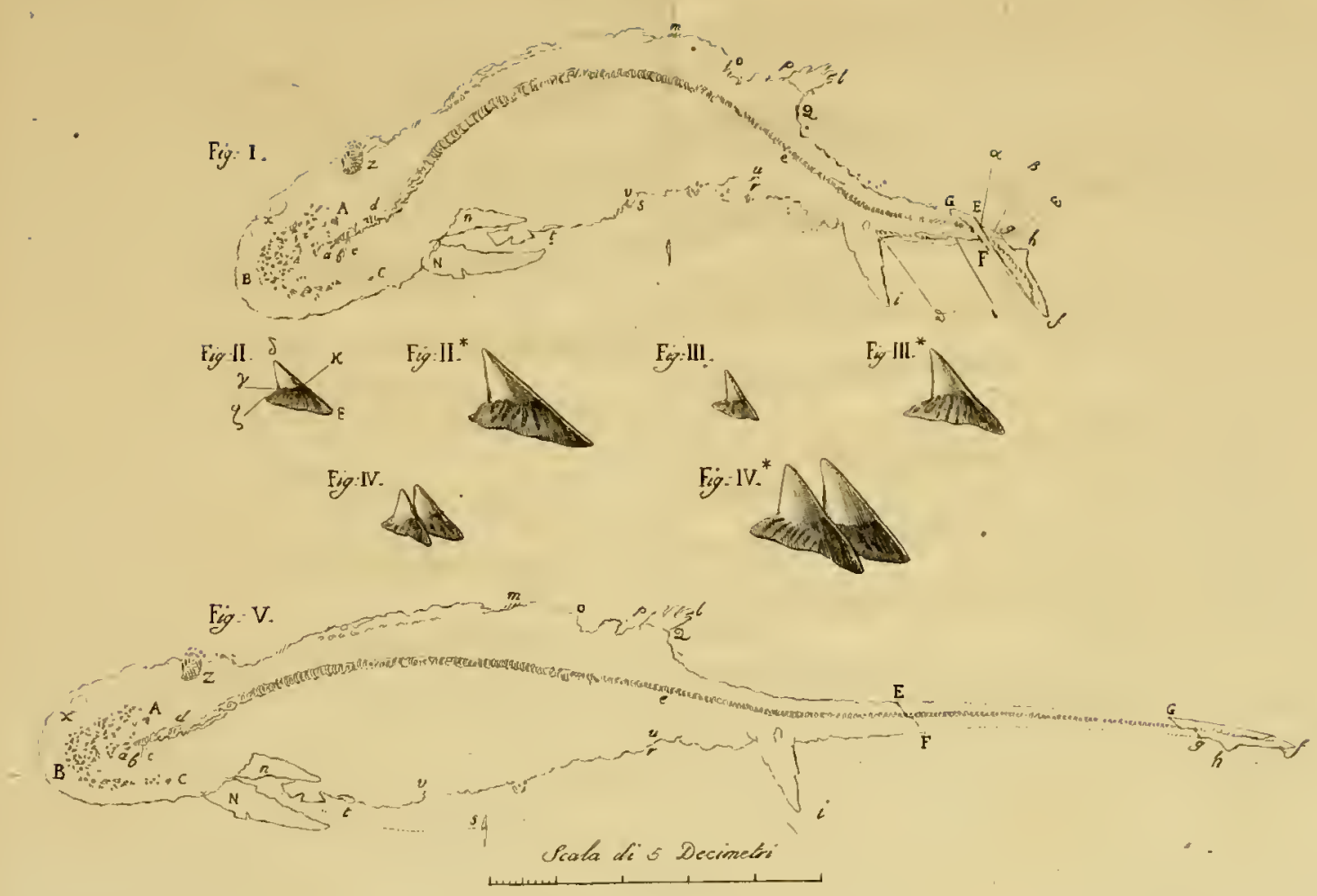






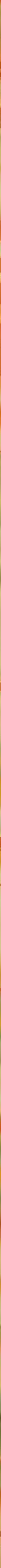


